\title{
Correspondence
}

\section{Grain boundary grooving in ice in a scanning electron microscope}

Recently, Barnes and Wolff (2004) claimed that the location of etch channels on the surface of a rapidly sublimating polycrystalline ice sample in a scanning electron microscope (SEM) does not coincide with the intersection of grain boundaries (GBs) with that surface. To support this, they showed a secondary electron (SE) image of surface etch channels that do not appear to intersect with the GBs seen on the concave surface of a nearby bubble. The purpose of this communication is to present an alternative explanation for this apparent lack of intersection between the GBs and etch channels in their example, to demonstrate, using thermodynamics, that the sublimation of etch channels vertically downwards, as described in their earlier work (Barnes and others, 2003), is unlikely, and to discuss the observation of GBs in ice that is sublimating.

The illusion of GB grooves on a concave (bubble) surface that do not intersect with their positions on the surrounding flat surface is typical of SE images produced using an Everhart-Thornley-type detector, in which nonline-of-sight SEs are attracted into the detector by its positive bias (Brandon and Kaplan, 1999). To illustrate this effect, we glued together two flat aluminum blocks and used a 1/8 in $(\sim 3 \mathrm{~mm})$ diameter ball end mill to produce hemispherical dimples of various depths along the joined surface. The sample was mounted in a JEOL 5310LV SEM with the surface seam perpendicular to the line of sight of the SE detector. Figure 1 shows the dimple horizontal, and tilted toward and away from the detector. When tilted, the intersection of the seam with the hemispherical surface appears not to be coincident with its location on the flat surface. Even in the horizontal position, the actual intersection at the outer radius of the dimple is hard to see, due to the sharp inclination of the side-wall of the dimple. By comparing SE images from shallow and deep dimples (Fig. 2) we see that it is precisely the slope of this side-wall that makes the seam appear to be non-continuous with the surface in the deeper dimple.

We believe that a similar phenomenon, i.e. visual distortion due to imaging a hemispherical surface with SEs, underlies the analysis of Barnes and others, and that their results do not contradict previous studies stating that grooves observed on an etched surface are associated with preferential sublimation at the GB (Cullen and Baker, 2001; Barnes and others, 2003).

A basic thermodynamic argument also supports this view. At a given temperature, the sublimation rate is a function of surface energy and vapor pressure, a relationship embodied in Kelvin's equation, derived by balancing the forces acting on a curved surface (Howe, 1997), i.e.

$$
\ln \left(P_{r} / P_{0}\right)=2 \gamma V_{\mathrm{m}} / R T r
$$

where, $P_{r}$ is the pressure above a surface of curvature, $r, P_{0}$ is the pressure above a flat surface, $\gamma$ is the surface energy, $V_{\mathrm{m}}$ is the molar volume of the phase, $R$ is the gas constant and $T$ is the temperature. The result is that a protrusion, or convex

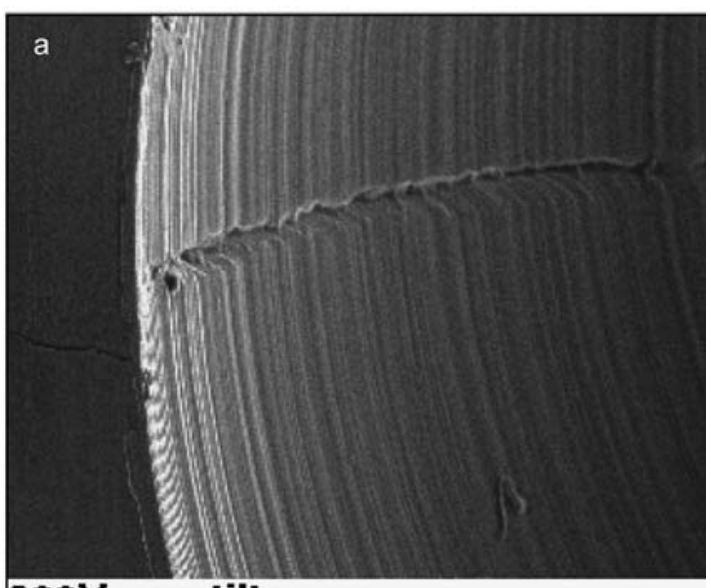

$200 \times$ max tilt away

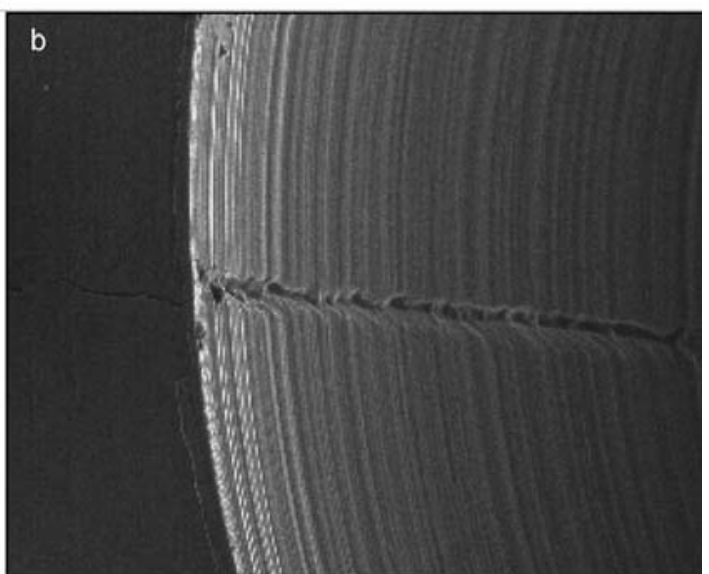

$200 \times$ level

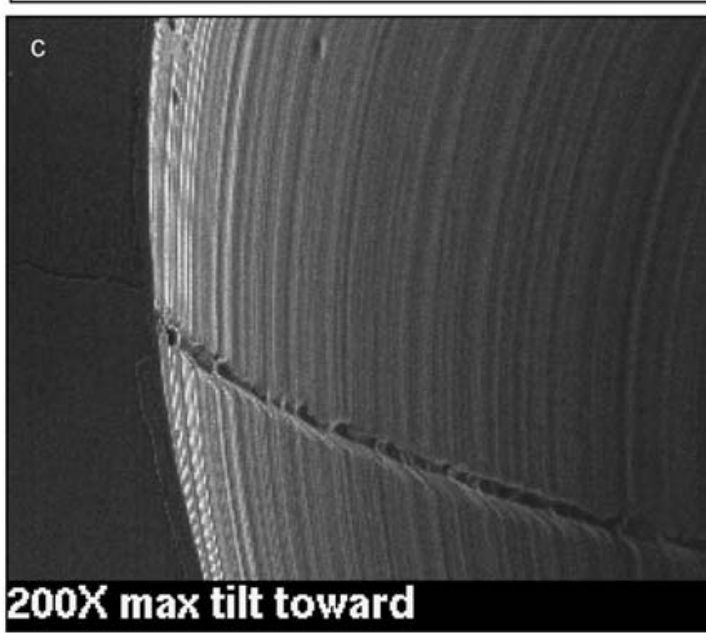

Fig. 1. Secondary electron images taken with an Everhart-Thornley detector, showing the intersection of a planar joint between two aluminum blocks with a concave surface produced by a ball end mill. In (a) and (c) the specimen stage is tilted from its normal horizontal position, away from and toward the detector, respectively. In (b) the stage is in its normal (horizontal) position. Even here it is not clear that the joint runs continuously through the specimen. 

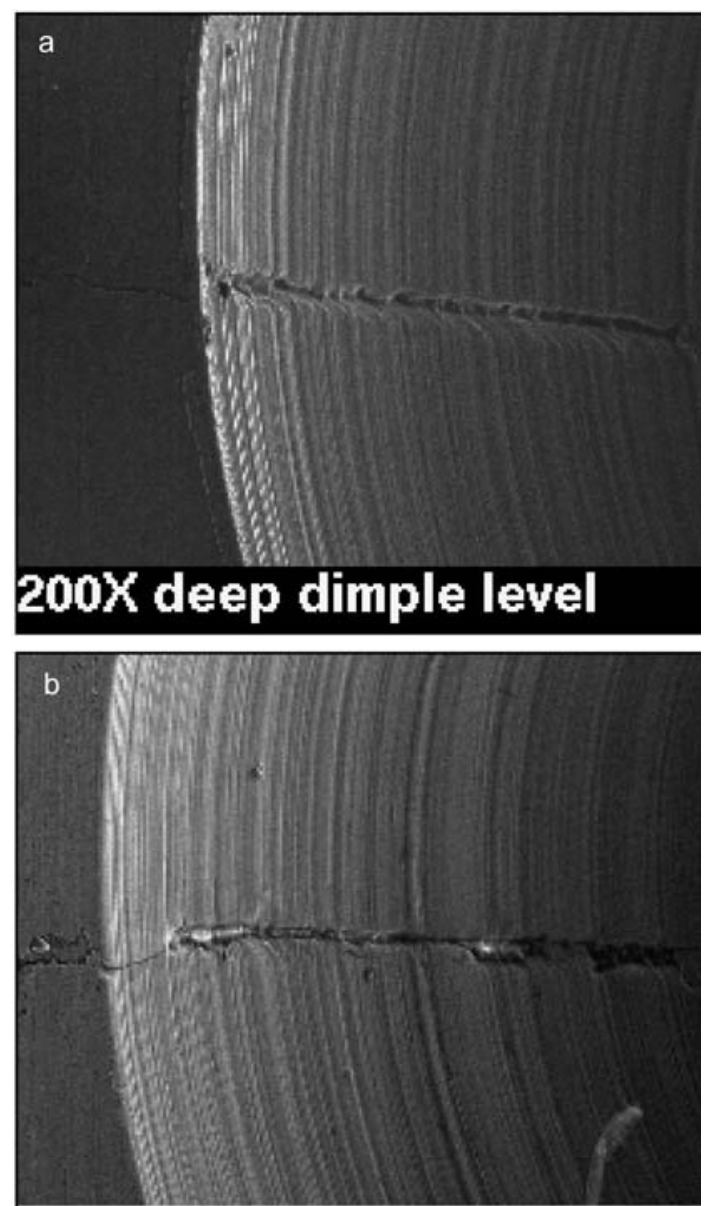

\section{$200 \mathrm{X}$ shallow dimple level}

Fig. 2. Images of (a) deep (1/16 in) and (b) shallow (1/32 in) dimples, produced as for Figure 1, illustrate that the steepness of the sidewalls contributes to the perception of discontinuity. Even with no stage tilt and the shallower dimple, the walls create the illusion that the joint is not planar.

surface, has a lower vapor pressure over it than over a flat surface, whereas a concavity has a higher vapor pressure than a flat surface. Based on this, we would expect a protrusion to sublimate more rapidly and a cavity to sublimate less rapidly than a flat surface.

It is also instructive to consider in detail the surface energy related to dangling bonds at the crystal-vapor interfaces. Porter and Easterling (1993) use a two-dimensional 'brokenbond' model based on the simple cubic crystal structure to illustrate the presence of additional dangling bonds on steps (macroscopic surfaces not parallel to a close-packed plane). A similar two-dimensional model is shown in Figure 3, where each lattice point is represented by a square with four nearest neighbors.

To remove an atom, we must break the bonds tying it to the surface. The squares on the close-packed surface $(P)$ have the most intact bonds and lowest surface energy. Square 1, on the protrusion, has the most dangling bonds and the highest surface energy. Thus, 1 is most likely to sublimate first. After 1 is removed, either of those labeled 2 has greater energy than the one between them and can more easily be removed. In this manner the protrusion will disappear. Next, we examine a channel. Here, square 3 and atoms on the sides of the channel have a higher surface

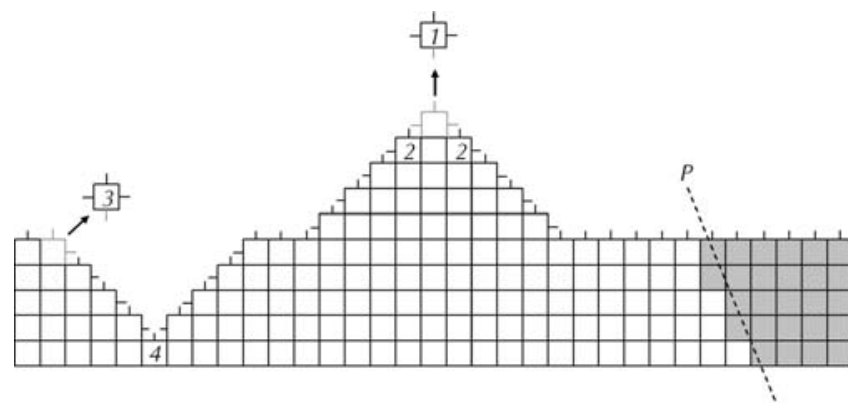

Fig. 3. The broken-bond model for surface energy of a crystal.

energy than the flat surface, whereas 4 has the same energy. Thus the channel is more likely to widen, and eventually disappear, than to deepen. The only thing that would cause the channel to continue to deepen would be the presence of more dangling bonds, i.e. a higher energy region, near its base.

A GB can be viewed as a sheet of higher-energy material, caused by the imperfect union of two differently oriented lattices, resulting in a region of unsatisfied bonds. Hence, where the GB intersects the surface it will sublimate preferentially to the areas on either side of the intersection, producing a GB groove or etch channel. If the $G B$ is not perpendicular to the specimen surface, the position of this channel will change during sublimation to follow the GB. Thus, when channels are seen on the surface of a specimen undergoing sublimation, as in Barnes and others (2003) and Barnes and Wolff (2004), they are more likely to be associated with the current location of a GB than with a groove left by its previous location. Taken together with our illustration earlier in this communication, it appears that the GB/etch channel mismatch shown in Barnes and others (2003) and Barnes and Wolff (2004) is an illusion.

This model also helps explain the shape of grain boundaries and the ease of their detection on a sublimating surface. The close-packed basal plane, and a macroscopic surface parallel to it, will sublimate most slowly. Prismatic, pyramidal or higher $\{h k l\}$ index planes will have more dangling bonds (see $P$ in Fig. 3), a higher surface energy and a faster sublimation rate. When we allow an ice specimen to sublimate in the SEM, we observe the emergence of etch patterns on the surface. As the water molecules detach themselves from the solid, preferentially from higher index planes, a series of steps are left on the surface. The resulting patterns can be used to identify differences in crystallographic orientation between grains and help to locate grain boundaries. Where two grains meet at the surface, the additional unsatisfied bonds at the grain boundary lead to greater sublimation and formation of the grain boundary channel. The relative orientations of the adjacent grains to the polycrystalline surface and to each other determines the shape of the grain boundary channel. We observe shallow troughs, symmetric and asymmetric V-shaped grooves, and stepped surfaces (examples shown in Fig. 4).

When only a small difference in orientation exists between adjacent grains, the grain boundary can be difficult to see, as in Figure 5, and its presence can be better detected with orientation imaging, such as electron backscatter diffraction. 

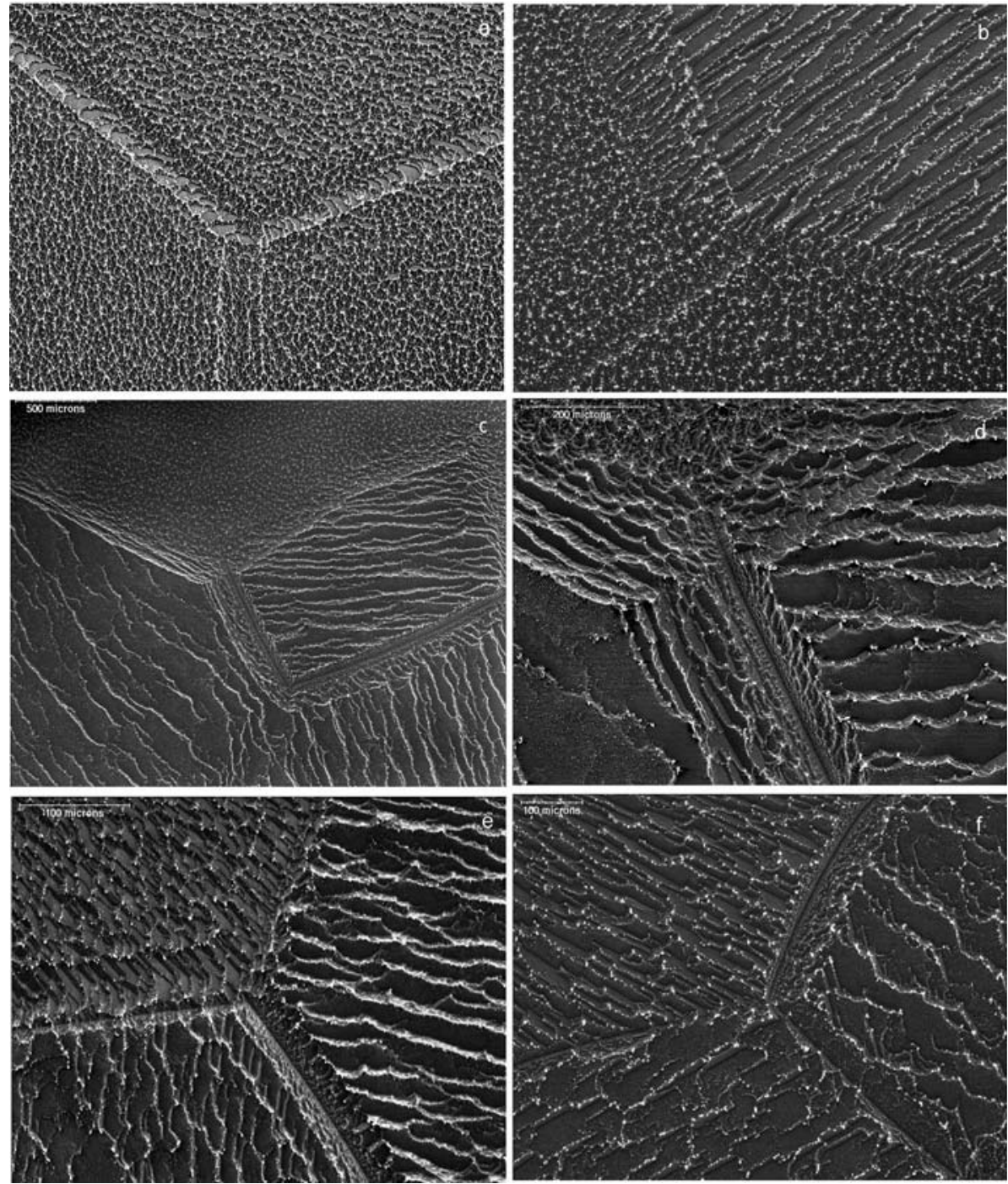

Fig. 4. Examples of SEM images of grain boundaries in polycrystalline ice.

Thus we conclude that attention to thermodynamic principles and an examination at the atomistic level of the behavior at the solid-vapor interface is necessary to interpret SEM images and provides the most logical, well-founded explanation of the phenomena we observe at the polycrystalline surface.

\section{ACKNOWLEDGEMENTS}

This research was supported by US Army Research Office (ARO) grant DAAD 19-03-1-0110 and US National Science Foundation (NSF) grants OPP-0221120 and 0440523. The views and conclusions contained herein are those of the authors and should not be interpreted as representing official policies, either expressed or implied, of the NSF, ARO or the United States Government.

Thayer School of Engineering,

Dartmouth College, Hanover,

New Hampshire 03755-8000, USA

E-mail: rachel.w.obbard@dartmouth.edu

Rachel OBBARD Ian BAKER

Daniel ILIESCU

\section{December 2005}

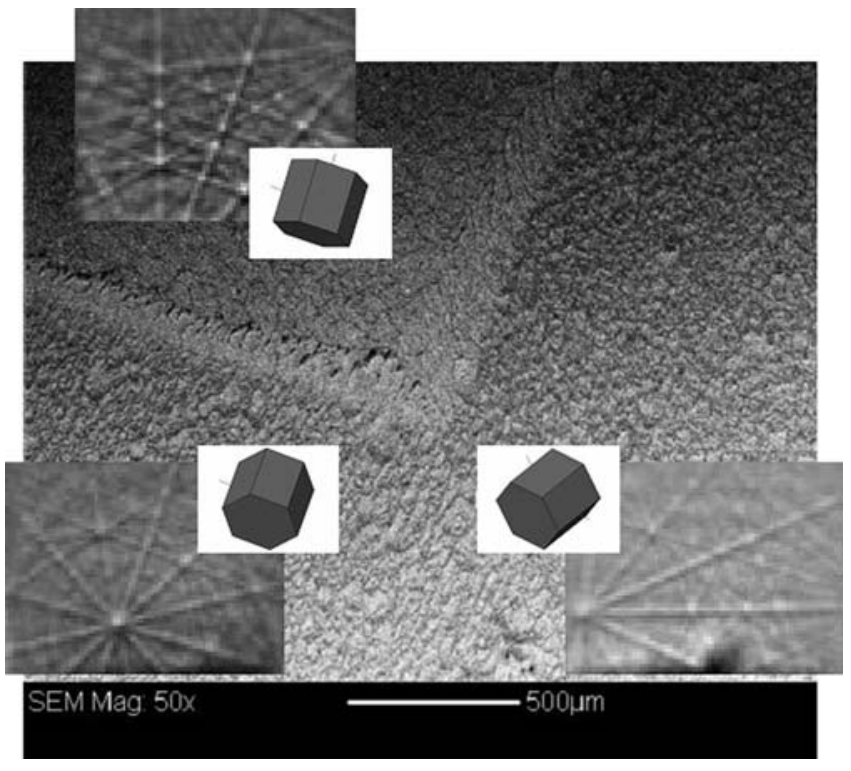

Fig. 5. Adjacent grains in Vostok (Antarctica) ice, and their associated orientations (analyzed with electron backscatter diffraction). Note that the grain boundary between the two grains at the bottom of the image is difficult to detect visually. 


\section{REFERENCES}

Barnes, P.R.F. and E.W. Wolff. 2004. Distribution of soluble impurities in cold glacial ice. J. Glaciol., 50(170), 311-324.

Barnes, P.R.F., E. Wolff, D.C. Mallard and H.M. Mader. 2003. SEM studies of the morphology and chemistry of polar ice. Microsc. Res. Techn. 62, 62-69.

Brandon, D.D. and W.D. Kaplan. 1999. Microstructural characterization of materials. New York, John Wiley.
Cullen, D. and I. Baker. 2001. Observation of impurities in ice. Microsc. Res. Techn. 55, 198-207.

Howe, J.M. 1997. Interfaces in materials: atomic structure, thermodynamics and kinetics of solid-vapor, solid-liquid and solid-solid interfaces. New York, John Wiley.

Porter, D.A. and K.E. Easterling. 1993. Phase transformations in metals and alloys. Second edition. London and New York, Chapman \& Hall. 